\title{
Transformational Leadership and Employee Innovation: A Moderated Mediating Effect Model
}

\author{
Caili Duan* \\ School of economics and management, Beijing Jiaotong University, Beijing, China \\ *Corresponding author. Email: 1109073876@qq.com
}

\begin{abstract}
How to motivate employees' innovative behavior is a constant concern of enterprise innovation management. At present, the research on the superior leadership and employee innovation behavior only reflects the direct relationship between the two, but the "black box" of the mechanism of the effect between the two has not yet been opened up. Based on individual motivation theory, this paper constructs a mediated model with regulation effect. The results show that transformational leadership is conducive to motivating employees' innovative behavior, and employees' psychological ownership plays a mediating role in the mechanism of the effect, and task complexity plays a regulatory role between psychological ownership and innovative behavior.
\end{abstract}

Keywords: Transformational leadership, Employee innovation behavior, Psychological ownership, Task complexity.

\section{INTRODUCTION}

There are different views on the role of transformational leadership [1] in employees' innovative behavior. One party believes that transformational leadership is the driving force of individual innovation behavior [2]. On the other hand, the negative relationship between the two was considered; the transformational leadership style inhibited the employee innovation behavior [3]. Thus, the existing research can not reach an agreement on the conclusions of the two variables, probably due to the existence of mediating or adjusting variables between the two, or the environment, culture, etc. in the study [4].

However, the literature that are related to transformational leadership and innovative behavior usually focus on the direct influence of the former on the latter, and the internal mechanism of the two is not clear enough. Therefore, the author uses the perspective of individual motivation to explore the internal influence mechanism of transformational leadership and employee's innovative behavior, and tries to open the black box between them. Task complexity is an important factor in regulating the relationship between leadership style and innovation, and task complexity affects employees' commitment to work [5]. But high task complexity also raises the risk of employee innovation.

\section{CONCEPTUAL FRAMEWORK AND RESEARCH HYPOTHESIS}

\subsection{The Conceptual Framework}

It is believed that the transformational leaders usually set up a harmonious atmosphere for communication with employees at work, so that employees feel encouraged and valued, and in return, to meet the expectations of the leader [6]. The study starts focusing on the intrinsic mechanism not the relationship on the surface between the two, and considers that the transformational leadership has an impact on the subordinate psychological level first and then uses the variable on the psychological level as a medium to predict the employee's innovative behavior [7]. As the outcome variable of leadership behavior and the antecedent variable of employee behavior, the psychological effect of psychological ownership on the mediating effect between the two variables has been confirmed in many literature. Therefore, we build an intermediary model with adjustment effect. The conceptual model is shown in Fig. 1. "PO" represents individual psychological ownership; "TC" represents 
task complexity; "IB" represents employee innovation; "TL" represents transformational leadership.

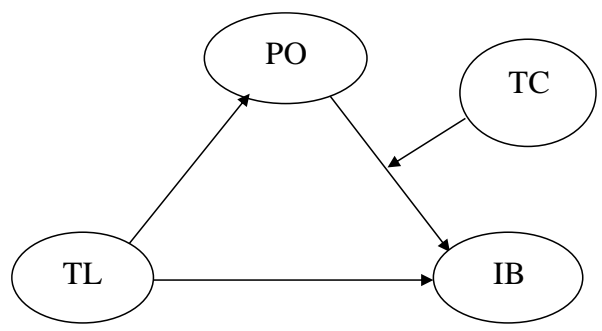

Figure 1 The conceptual model.

\subsection{The Research Hypothesis}

\subsubsection{The Effect of Transformational Leadership on Innovative Behavior}

Transformational leaders encourage employees to look at problems critically, and ultimately lead employees to innovate [8]. Later, it is proved that transformational leadership has an impact on the psychological state of subordinates, and then affects its innovative behavior. Transformational leaders tend to build a organizational vision toward their subordinates, make them more explicit and devote more energy to the organizational innovation goals, resulting in innovative behavior [9].

Therefore, we propose the hypothesis,

H1: Transformational leadership is conducive to employee innovation.

\subsubsection{The Relationship between Psychological Ownership and Innovative Behavior}

Psychological ownership is proposed by Pierce et al. [10], which represents the individual's feelings of ownership of the target. Organizational ownership of emotions will give employees more sense of responsibility to the organization, and make them more active toward the development of the organization [11]. Second, employees' emotional ownership of their work motivated them to take more responsibility for the job and self-efficacy of psychological ownership enables employees to have more confidence in fulfilling their tasks and goals [12]. Therefore, the enhancement of individual psychological ownership will exert favorable influence on the emergence of innovative behavior.

We can get the hypothesis,

H2: Employees' psychological ownership can significantly predict their innovative behavior.

\subsubsection{The Relationship between}

Transformational Leadership and Psychological Ownership

The root causes of psychological ownership come mainly from the individual's satisfaction with the needs: sense of space, self-efficacy and self-identity [13]. Therefore, the psychological ownership can first be generated through the control of the individual on things, or through the relationship between the individual and the target, and finally, as individuals invest more in their goals, his has deeper ownership. Among several typical leadership styles, workers under transformational leadership have the strongest psychological ownership of the organization [14]. The transformational leader is more inclined to give subordinates authorization in his work., so his sense of ownership gradually increases; second, the transformational leadership creates an atmosphere of encouragement and harmony with employees, promotes the continuous transformation of employees' roles toward leadership [15] thereby enhancing employee self-efficacy. Third, the open communication and the personal care to subordinates will lead to a sense of belonging and intimacy. It can be seen that the psychological effect of transformational leadership on subordinates can enhance psychological ownership of subordinates.

So, we propose the hypothesis,

H3: Transformational leadership can predict subordinate psychological ownership.

\subsubsection{Intermediary Role of Psychological Ownership}

Previous literature prove that psychological ownership can act as an intermediary variable that affects individual behavior. The three types of variables: repositioning of psychological cognition, reinterpretation of the working environment and evaluation of social relations usually play an intermediary role in transformational leadership and dependent variables. And psychological ownership is the repositioning of individual psychological cognition, therefore, this study supposes that transformational leadership may motivate subordinates to behave favorably toward the organization through psychological ownership.

Therefore, it is hypothesized that,

H4: Psychological ownership plays an intermediary role in the model.

\subsubsection{The Regulating Effect of Task Complexity}

Task characteristics, as one of the most important team situational variables, play an important role in the 
formation of employees and can significantly promote or hinder them. Task complexity refers to the complexity of the work task structure, which is usually characterized by multiple goals, multiple paths and multiple conflicts. A certain degree of complexity can encourage employees to learn new methods and technologies, thus promoting the generation of innovative behaviors. The more complex the task, the more likely employees are to think creatively [16]. And Task complexity is an important factor in regulating the relationship between leadership style and innovation, and task complexity affects employees' commitment to work [5].

Therefore, we put forward the hypothesis that,

H5: Task complexity regulates the relationship between psychological ownership and innovation.

\section{THE RESEARCH DESIGN}

We adopted the methods of investigation and research, including construction and measurement, data collection (Pre-survey, formal investigation), reliability and validity test, construct discrimination analysis, and statistics and analysis using SPSS19.0 and Amos17.0 statistical software.

\subsection{Data Collection}

The research takes the employees in North China as the main survey object and conducts the questionnaire survey. The study's survey began on August 10, 2020 for a total of 30 days. A total of 300 questionnaires were sent out. Finally, 217 valid questionnaires were received, and the effective rate of the questionnaire was $72.3 \%$. The proportions of the sample gender, education level, length of service, function distribution, job level, and the nature of the unit are basically the same, and proportion of people aged 25-30 years is 60.4\%, and that of $31-40$ years is $39.6 \%$.

\subsection{Construction and Measurement}

The scales used in the study are widely recognized and applied at home and abroad. For the measurement of IB, we select the scale of Scott \& Bruce and the Cronbach's coefficient is 0.873 . For the measurement of TL, we select the scale of Li Chaoping \& Shi Kan [17], and the Cronbach's coefficient is 0.934 . For the

Measurement of PO, we select the scale of Avey etc. [18], and the Cronbach's coefficient is 0.952 . For the measurement of TC, we select the scale of Zhao Xiping and Kong Fang [19], and the Cronbach's coefficient is 0.971. In this paper, the Cronbach's coefficient of the scale is greater than 0.8 , so the scale has a good reliability, which means that the data can be further analyzed. Confirmatory factor analysis shows that the measurement model has a good fit $\left(\lambda^{2} / \mathrm{df}=2.157\right.$, RMSEA=0.069, GFI=0.923, CFI=0.951, NFI=0.924, IFI=0.933).3.3. Data Analysis and Results

\subsubsection{Descriptive Statistics Analysis and Correlation Analysis}

In the process of data analysis, three statistical variables that may affect the behavior and perception of subjects are used as control variables. The mean, standard deviation and correlation coefficient of each variable are as follows. Table 1 shows that the correlation coefficient between variables is significant ("***" means $\mathrm{p}<0.001$, "**" means $\mathrm{p}<0.01$ and "*" means $\mathrm{p}<0.05)$.

\subsubsection{Hypothesis Test}

The regression analysis of $\mathrm{IB}, \mathrm{PO}$ and TL was made, as shown in "table 2". In Model 2, $\beta=0.382, \mathrm{P}<0.001$, this indicates that the style of the leader is closer to the transformational leadership; the more individuals tend to show innovative behavior, so hypothesis $\mathbf{H 1}$ is valid.

In Model 3, $\beta=0.505, P<0.001$, indicating that the higher the individual's psychological ownership, the more inclined to show innovative behavior, hypothesis $\mathbf{H} 2$ is valid.

In Model $1, \beta=0.350, \mathrm{P}<0.05$, indicating that the level of individual psychological ownership will increase with the improvement of transformational leadership, hypothesis $\mathbf{H 3}$ is valid.

In this paper, the method of testing the mediating role of PO is mainly referred to the mediator model test method of Wen Zhonglin. [20] Based on Model 3, we introduce psychological ownership in Model 4, compared with Model 3, R2 in Model 4 changes significantly, and $\beta=0.628, P<0.001$. The coefficient of the intermediate variable PO was significant (as shown in Model 4 in "table 2"), and the coefficient of the intermediate variable TL was not significant, so, psychological ownership plays a completely intermediary role, $\mathbf{H 4}$ is assumed to be true.

Further, compared to Model 5 and Model 3 ("table $2 ")$, As we know from Model 5 , the $\mathrm{R}^{2}$ of the model changes significantly after the introduction of task complexity, and the regression coefficient of the regulatory effect is, $\beta=0.177, \quad \mathrm{P}<0.001$, so, task complexity can regulate the relationship between psychological ownership and employee innovation, then the hypothesis $\mathbf{H 5}$ is verified. From model 6, it can be seen that the $\mathrm{R}^{2}$ of the model increases significantly, and the coefficients of main effect, mediating effect and regulating effect are significant, therefore, the model in this paper is valid. 
Table 1. Mean standard deviation and correlation coefficient of the variables

\begin{tabular}{|c|c|c|c|c|c|c|c|c|}
\hline Variables & Mean & Standard Deviation & 1 & 2 & 3 & 4 & 5 & 6 \\
\hline Berufsgruppe & 2.78 & 1.406 & & & & & & \\
\hline Position Level & 1.81 & 0.869 & 0.519 & & & & & \\
\hline Nature of Unit & 1.73 & 0.677 & -0.583 & -0.300 & & & & \\
\hline IB & 4.093 & 1.077 & 0.131 & -0.032 & -0.249 & & & \\
\hline TL & 3.446 & 0.754 & -0.009 & 0.039 & -0.202 & $0.398^{\star \star}$ & & \\
\hline PO & 4.075 & 0.902 & -0.013 & -0.014 & -0.167 & $0.525^{\star *}$ & $0.338^{\star \star}$ & \\
\hline TC & 4.713 & 1.048 & 0.081 & 0.023 & 0.089 & $0.482^{\star \star}$ & $-0.264^{\star \star}$ & -0.065 \\
\hline
\end{tabular}

Table 2. Regression analysis results

\begin{tabular}{|c|c|c|c|c|c|c|}
\hline \multirow{2}{*}{ Variables } & \multirow{2}{*}{$\frac{\mathrm{PO}}{\text { Model } 1}$} & \multicolumn{5}{|c|}{ IB } \\
\hline & & Model 2 & Model 3 & Model 4 & Model 5 & Model 6 \\
\hline Berufsgruppe & -0.061 & 0.150 & 0.133 & 0.112 & -0.042 & -0.019 \\
\hline position level & -0.053 & -0.170 & -0.145 & -0.137 & -0.077 & -0.088 \\
\hline Nature of unit & 0.036 & -0.121 & -0.122 & -0.144 & -0.244 & -0.220 \\
\hline $\mathrm{TL}$ & $0.350^{*}$ & $0.382^{* \star *}$ & & -0.152 & & $0.226^{\star *}$ \\
\hline $\mathrm{PO}$ & & & $0.505^{\star \star \star}$ & $0.628^{\star * *}$ & $0.321^{\star \star *}$ & $0.339 * * *$ \\
\hline $\mathrm{TC}$ & & & & & $0.229^{* * *}$ & $0.274^{\star * *}$ \\
\hline $\mathrm{PO} * \mathrm{TC}$ & & & & & $0.177^{\star * \star}$ & $0.170^{\star \star *}$ \\
\hline $\operatorname{adj} R^{2}$ & 0.190 & 0.203 & 0.315 & 0.318 & 0.326 & 0.331 \\
\hline
\end{tabular}

\section{CONCLUSION}

Transformational leadership influences employees' innovation behavior through the mediating effect of employees' psychological ownership. So transformational leadership can induce individuals to produce innovative behavior, but the relationship between the two is not a direct causal relationship. The creative behavior of employees is determined by individual motivation. In particular, psychological ownership is the medium through which the transformational leadership promotes the individual innovation behavior; transformational leadership inspires individual innovation motivation by changing the level of individual psychological ownership, thus showing innovative behavior. Task complexity plays a moderating role in the mechanism of transformational leadership influencing employee innovation. The higher the task complexity, the more obvious the effect of employees' psychological ownership on innovation. Therefore, transformational leadership is the decisive organizational factor to induce individual innovation behavior, and task complexity determines the effect of transformational leadership and psychological ownership on employees' innovative behaviors.

\section{AUTHORS' CONTRIBUTIONS}

Through literature comparison, the author finds the research gap between transformational leadership and employee innovation, proves the moderating effect of task complexity and the mediating effect of psychological ownership, and develops the leadership theory and innovation theory.

\section{ACKNOWLEDGMENTS}

This research was financially supported by the The Fundamental Research Funds for the Central Universities (2019 YJS067).

\section{REFERENCES}

[1] J.M. Burns, Leadership, Harper\&Row, New York, 1978.

[2] Zhang Jun, Wang Guo-hong. Analysis on the Influence mechanism of transformational leadership on team Innovation Performance, Enterprise economy. 2020, (9), pp. 37-43. DOI: 10.13529/j.cnki.enterprise.economy.2020.09.005

[3] R. Basu, S.G. Green. Leader-member exchange and transformational leadership: An empirical examination of innovative behaviors in leader- 
member dyads, Journal of Applied Social Psychology. 1997, 27(6), pp. 477-499. DOI: 10.1111/j.1559-1816.1997.tb00643.x

[4] Chen Chen, Shi Kan, Lu Jiafang. Transformational leadership for creative behavior: A moderated mediation effect model, Journal of Management Science. 2015, (4), pp. 11-22. DOI: 10.3969/j.issn.1672-0334.2015.04.002

[5] Zhao Jia, Luo Jinlian. The Influence of Shared leadership of R\&D Team on Creativity -- An Adjustable intermediary Model, Research in Technology Economy and Management. 2020, (9), pp. 51-56.

[6] Ding Lin, Xi Youmin, Zhang Hua. Transfornational leadershp and employee creativity: The mediating role of supervisor-subordinate relationship, Science Research Management. 2010, 31(1), pp. 178-179. DOI: 10.19571/j.cnki.1000-2995.2010.01.022

[7] Liu Jingjiang, Zou Huimin. The impact of transformational leadership and psychological empowerment on employee creativity, Science Researcli Management. 2013, (3), pp. 68-74. DOI: 10.19571/j.cnki.1000-2995.2013.03.009

[8] L. Furby. Possession in humans: An exploratory study of its meaning and motivation, Social Behavior and Personality. 1978, 6(1), pp. 49-65. DOI: $10.2224 / \mathrm{sbp} .1978 .6 .1 .49$

[9] D.I. Jung, C. Chow, A. Wu. The role of transformational leadership in enhancing organizational innovation-hypotheses and some preliminary findings, The Leadership Quarterly, 2003, (14), pp. 525-544. DOI: 10.1016/S10489843(03)00050-X

[10] J.L. Pierce, S.A. Rubenfel, Morgans. Employee ownership: A conceptual model of process and effects, Academy of Management Review. 1991, 16(2), pp. 121-144.

[11]P. Tierney. S. Farmer. The PyGmalion process and employee creativity, Journal of Management. 2004, 30(3), pp. 413-432. DOI: 10.1016/j.jm.2002.12.001
[12] J.L. Pierce, T. Kostova, K.T. Dirks. Toward a theory of psychological ownership in organizations, Academy of Management Review. 2001, 26(2), pp. 298-310. DOI: $10.2307 / 259124$

[13] Chen Hao. Research on the impact of leadership style on employee's psychological ownership, Technical Economy and Management Research. 2011, (11), pp. 54-57. DOI: 10.3969/j.issn.1004292X.2011.11.013

[14] R.W. Woodman, J.E. Sawyer, R. Griffin. Toward a theory of organizational creativity, Academy of management Review. 1993, 18(2), pp. 293-321.

[15]Zhang Xiaoyi, Wang Yunfeng, Yu Wei. Focused climate: A literature review and prospects, Foreign Economics \& Management. 2016, 38(9), pp. 64-66. DOI: $10.16538 /$ j.cnki.fem.2016.09.005

[16] Shu Qian. Research on the Impact of Task complexity on Employee innovation Behavior -The Moderating Effect of Core SelfEvaluation,Time Finance. 2020, (6), pp. 113-114. DOI:CNKI:SUN:YNJR.0.2020-06-055

[17]Li Chaoping, Shi Kan. The structure and measuranent of transformational leadership in China, Acta Psychologica Sinica. 2005, 37(6), pp. 803-811. DOI: 10.1007/s11782-008-0032-5

[18] J.B. Avey, B. Avolio, C. Crossley. Psychological ownership: Theoretical extensions, measurement and relation to work outcomes, Journal of Organizational Behavior. 2009, 30(2), pp. 173-191. DOI: $10.1002 /$ job.583

[19]Zhao Xiping, Kong Fang. Self-efficacy and threedimensional performance of researchers: The Moderating Effect of Work Complexity, Soft Science. 2011, 25(2), pp. 104-107. DOI: 10.3969/j.issn.1001-8409.2011.02.022

[20] Wen Zhonglin, Ye Baojuan. Different methods for testing moderated mediation models: Competitors or backups, Acta Psychologica Sinica. 2014, 46(5), pp. 714-726. DOI: 10.3724/SP.J.1041.2014.00714 